NUNES, G.H.S.; SANTOS JÚNIOR, J.J.S.; ANDRADE, F.V.; BEZERRA NETO, F.; ALMEIDA, A.H.B.; MEDEIROS, D.C. Aspectos produtivos e de qualidade de híbridos de melão cultivados no agropolo Mossoró-Assu. Horticultura Brasileira, Brasília, v.22, n.4, p.744-747, out-dez 2004.

\title{
Aspectos produtivos e de qualidade de híbridos de melão cultivados no agropolo Mossoró-Assu
}

\author{
Glauber Henrique de S. Nunes; João José dos Santos Júnior; Fábia V. Andrade; Francisco Bezerra Neto; \\ Antônio Hélio B. de Almeida; Damiana Cleuma de Medeiros \\ ESAM, C. postal 137, 59.625-900 Mossoró-RN; E-mail: glauber@esam.br.
}

\begin{abstract}
RESUMO
Dois experimentos foram realizados com o objetivo de avaliar o desempenho produtivo e qualitativo de híbridos de melão no agropolo Mossoró-Assu. Em ambos os experimentos foi utilizado o delineamento em blocos casualizados com quatro repetições. O primeiro experimento foi constituído de seis híbridos de melão Gália e dois do tipo Cantaloupe, e o segundo foi composto de sete híbridos de melão Amarelo, um Pele de sapo e três Orange Flesh. Avaliaram-se a produtividade, peso médio do fruto, cavidade interna, espessura da polpa, aparências externa e interna, perda de peso, teor de sólidos solúveis e firmeza da polpa. Considerando as variáveis utilizadas na avaliação, constatou-se variação entre os híbridos nos dois experimentos. Entre os melões Gália, destacaram-se os híbridos DRG 1531 e DRG 1537. Os melões Red Flesh e AFX 700 foram os mais promissores entre os melões do tipo Orange Flesh. Por fim, entre os melões do tipo Valenciano, destacaram-se os híbridos Gold Mine, Gold Pride e Gold Star.
\end{abstract}

Palavras-chave: Cucumis melo L., qualidade, produtividade, armazenamento.

\begin{abstract}
Yield and quality aspects of melon hybrids grown in agropolo Mossoró-Assu

Two experiments were carried out with the objective of evaluating the quality and yield performance of hybrids of melon in agropolo Mossoró-Assu, Brazil. In both experiments a randomized complete blocks design with four replications was used. In the first experiment six Galia and two Cantaloupe hybrids were used and in the second experiment seven Yellow hybrids, one Piel del Sapo and three Orange Flesh hybrids were evaluated. Evaluations of productivity, average fruit weight, internal cavity, pulp thickness, external and internal appearance, weight loss, total soluble solids and pulp firmness were made. Variation among the hybrids in both experiments for the assessed characteristics were observed. Among Gália melons, the hybrids DRG 1531 and DRG 1537 were the most promising while among Orange Flesh melons, the Red Flesh and AFX 700 hybrids were the best. Among Yellow melons, the hybrids Gold Mine, Gold Pride and Gold Star were the most promising.
\end{abstract}

Keywords: Cucumis melo L., quality, yield, storage.

(Recebido para publicação em 17 de fevereiro de 2004 e aceito em 27 de outubro de 2004)

$\mathrm{O}$ melão (Cucumis melo L.) é a olerícola de maior destaque no Rio Grande do Norte (Silva, 1999). Em 1998, as 58.900 toneladas exportadas deram ao Rio Grande do Norte o título de líder nacional, com $91 \%$ de participação no mercado, com um aumento de 34,2\% em volume em relação a 1996 (Menezes et al., 2001a). O emprego de alta tecnologia, associado às condições ótimas de clima para o seu desenvolvimento, têm permitido o aumento contínuo das exportações de melão nos últimos anos.

As cultivares do grupo Inodorus, representadas pelo tipo Amarelo, Pele de Sapo e Orange Flesh, são os preferidos pelos produtores, totalizando cerca de $90 \%$ da área plantada. Essa preferência é justificada pela excelente vida útil pós-colheita, em torno de 35 dias em condições ambiente (Nascimento, 2001). No entanto, recentemente, tem havido interesse em diversificar o pro- duto a ser oferecido para os mercados interno e externo pela introdução de diversos genótipos de melão, em especial, dos grupos Reticulatus e Cantaloupensis e do tipo Gália.

Em razão do interesse crescente por novos tipos de melão, as empresas sementeiras têm lançado, anualmente grande número de novos híbridos. Não obstante, a adoção de qualquer um desses híbridos sem uma prévia avaliação pode acarretar prejuízos na produtividade e qualidade do produto. Assim sendo, o conhecimento sobre a produção e o comportamento pós-colheita de novos materiais são fundamentais para que o produtor possa decidir, com segurança, pelo genótipo mais adequado para o cultivo.

O presente trabalho teve como objetivo avaliar, de forma preliminar, híbridos de melão quanto a produtividade e qualidade no agropolo Mossoró-Assu.

\section{MATERIAL E MÉTODOS}

$\mathrm{O}$ experimento foi instalado em Mossoró, município situado a aproximadamente $18 \mathrm{~m}$ de altitude, com coordenadas geográficas $5^{\circ} 11^{\prime}$ de latitude Sul e $37^{\circ} 20^{\prime}$ de longitude Oeste do meridiano de Greenwich. O clima, segundo a classificação de Koppen, é 'BSWh' (muito seco, com estação de chuva no verão atrasando-se para o outono).

No primeiro experimento foram avaliados os híbridos GPS 400, DRG 1531, DRG 1537, Supra, Galileu, Solarbel, todos melões Gália, além dos híbridos Cantaloupe, Hy Mark e Imperial. No segundo, foram utilizados os híbridos amarelos Rochedo, Gold Mine, Gold Pride, Gold Star, AFX 200, Amarillo CP e Canarian. O híbrido Tendency é do tipo Pele de sapo e os híbridos Saturno, Red Flesh e AFX 700 são do tipo Orange Flesh. 
Tabela 1. Médias das características produtividade (PROD), peso médio do fruto (PM), cavidade interna (CI), espessura da polpa (EP), Aparências externa (AE) e interna (AI), perda de peso (PP), sólidos solúveis totais (SST) e firmeza da polpa (FP) de híbridos de melão cultivados no agropolo Mossoró-Assu, Experimento 1. Mossoró, ESAM, 2002.

\begin{tabular}{lccccccccc}
\hline Híbrido & $\begin{array}{c}\text { PROD } \\
\text { (Mg/ha) }\end{array}$ & PM (g) & Cl (cm) & EP (cm) & AE & Al & PP (\%) & SSTI (\%) & FP (N) \\
\hline GPS 400 & $23,26 \mathrm{~b}$ & $1654 \mathrm{a}$ & $5,87 \mathrm{~b}$ & $2,80 \mathrm{~b}$ & $2,83 \mathrm{~b}$ & $3,42 \mathrm{~b}$ & $6,48 \mathrm{a}$ & $7,21 \mathrm{c}$ & $18,40 \mathrm{c}$ \\
Solarbel & $24,33 \mathrm{~b}$ & $1562 \mathrm{a}$ & $5,63 \mathrm{~b}$ & $3,38 \mathrm{a}$ & $4,00 \mathrm{a}$ & $4,00 \mathrm{a}$ & $5,52 \mathrm{a}$ & $7,02 \mathrm{c}$ & $26,10 \mathrm{~b}$ \\
Galileo & $23,65 \mathrm{~b}$ & $1652 \mathrm{a}$ & $6,12 \mathrm{~b}$ & $2,75 \mathrm{~b}$ & $4,00 \mathrm{a}$ & $4,50 \mathrm{a}$ & $6,17 \mathrm{a}$ & $8,43 \mathrm{~b}$ & $33,53 \mathrm{a}$ \\
DRG 1531 & $30,25 \mathrm{a}$ & $1354 \mathrm{a}$ & $5,44 \mathrm{~b}$ & $2,64 \mathrm{~b}$ & $3,17 \mathrm{~b}$ & $4,00 \mathrm{a}$ & $2,12 \mathrm{~d}$ & $9,81 \mathrm{a}$ & $35,25 \mathrm{a}$ \\
DRG 1537 & $31,25 \mathrm{a}$ & $1652 \mathrm{a}$ & $5,77 \mathrm{~b}$ & $2,55 \mathrm{~b}$ & $2,42 \mathrm{~b}$ & $5,00 \mathrm{a}$ & $3,38 \mathrm{c}$ & $10,25 \mathrm{a}$ & $33,28 \mathrm{a}$ \\
Supra & $32,54 \mathrm{a}$ & $1836 \mathrm{a}$ & $6,84 \mathrm{a}$ & $3,04 \mathrm{a}$ & $3,17 \mathrm{~b}$ & $4,58 \mathrm{a}$ & $4,26 \mathrm{~b}$ & $8,28 \mathrm{~b}$ & $21,85 \mathrm{~b}$ \\
Hy Mark & $31,59 \mathrm{a}$ & $1758 \mathrm{a}$ & $5,91 \mathrm{~b}$ & $3,49 \mathrm{a}$ & $3,44 \mathrm{a}$ & $3,50 \mathrm{~b}$ & $6,85 \mathrm{a}$ & $7,85 \mathrm{~b}$ & $20,35 \mathrm{~b}$ \\
Imperial & $18,25 \mathrm{c}$ & $1125 \mathrm{~b}$ & $5,66 \mathrm{~b}$ & $2,36 \mathrm{~b}$ & $1,00 \mathrm{c}$ & $2,58 \mathrm{~b}$ & $6,82 \mathrm{a}$ & $4,81 \mathrm{~d}$ & $15,13 \mathrm{c}$ \\
\hline Média & 26,89 & 1574,13 & 5,91 & 2,88 & 3,00 & 3,95 & 5,20 & 7,96 & 25,49 \\
CV\% & 32,39 & 18,04 & 3,42 & 11,12 & 18,93 & 17,86 & 25,40 & 8,48 & 10,49 \\
\hline
\end{tabular}

* Médias seguidas pela mesma letra nas colunas, não diferem, entre si, pelo teste de Scott-Knott, a 5\% de probabilidade.

O delineamento experimental utilizado foi o de blocos completos casualizados com quatro repetições. A parcela foi constituída por quatro linhas de 5 metros de comprimento, contendo 20 plantas cada, sendo que a área útil da parcela correspondeu as duas fileiras centrais. O espaçamento foi de 2,0x0,5 $\mathrm{m}$, com duas plantas por gotejador.

O preparo do solo e a correção do sistema de irrigação foram realizados de acordo com o sistema de produção local. Utilizou-se irrigação por gotejamento, com fertirrigação e os fertilizantes foram aplicados com base nas análises químicas de amostra do solo. As demais práticas culturais e fitossanitárias obedeceram às necessidades da cultura no estado.

Foram selecionados 12 frutos de cada parcela e transportados ao laboratório de pós-colheita da ESAM, e armazenados a $5^{\circ} \mathrm{C}$ (Gália, Charentais e Cantaloupe) e $8^{\circ} \mathrm{C}$ (Amarelo, Pele de Sapo e Orange Flesh).

As características avaliadas foram: produtividade ( $\mathrm{t} / \mathrm{ha}$ ); peso médio do fruto $(\mathrm{g})$, cavidade interna $(\mathrm{cm})$, espessura da polpa $(\mathrm{cm})$, teor de sólidos solúveis $(\%)$, firmeza da polpa $(\mathrm{N})$, perda de peso (\%) após vinte dias de armazenamento; aparências externa (depressões e lesões fúngicas) e interna (sementes soltas e presença de líquido), realizada utilizando-se uma escala visual e subjetiva, no final do armazenamento. A escala corresponde a notas variando de 1 a 5 , de acordo com a severidade dos defeitos (nota $1=$ defeitos extremamente se- veros em mais de $50 \%$ da área do fruto; nota $2=$ defeitos severos em 31 a $50 \%$ da área do fruto; nota $3=$ defeitos moderados em 11 a $30 \%$ da área do fruto; nota $4=$ defeitos leves em 1 a $10 \%$ da área do fruto e, nota 5= ausência de defeitos). Considerou-se como fruto inadequado para o consumo aquele cuja nota apresentou valor inferior a 3,0 (Gomes et al. , 2001). Os dados foram analisados por meio de análise de variância e aplicação do teste de agrupamento de Scott-Knott com 5\% de probabilidade para comparação múltipla.

\section{RESULTADOS E DISCUSSÃO}

No experimento 1, observou-se que os híbridos DRG 1531, DRG 1537, Supra e Hy Mark foram os mais produtivos. O híbrido Imperial foi o de menor produtividade. Uma explicação desse comportamento inferior desse híbrido foi ataque severo do fungo didymella bryoniae. Como somente as parcelas com esse híbrido estavam afetadas pelo fungo, suspeitou-se que as sementes estavam infectadas, fato comprovado posteriormente (Tabela 1).

Os híbridos AFX 200, Saturno, Red Flesh e AFX 700 foram os mais produtivos no experimento 2. A menor produtividade foi constatada no híbrido Amarillo CP. Ressalta-se que, dos quatro híbridos mais produtivos, três eram do tipo Orange Flesh (Tabela 2).

Segundo Dias (1998), a produtividade média de melão no Nordeste está entre 17 e 30 toneladas. Assim sendo, nos dois experimentos, a maioria das produtividades dos híbridos estavam dentro desse intervalo. As estimativas médias de todos os híbridos de destaque nos dois experimentos estavam acima de 30 t/ha.

Formaram-se dois grupos de híbridos quanto ao peso médio do fruto no primeiro experimento, sendo os genótipos DRG 1531 e Imperial aqueles de menor peso. No segundo experimento, houve maior variação entre os híbridos, com destaque para o híbrido Tendency.

O peso médio do fruto está diretamente relacionado com o tamanho do fruto. No mercado exterior, a preferência é por frutos de menor tamanho. Os melões avaliados estão dentro do peso médio dos frutos exportados (Soares, 2001). Por outro lado, os melões pele de sapo, com maior tamanho, são os preferidos na Espanha. Aproximadamente 9,29\% do melão exportado pelo porto de Natal é do tipo pele de sapo (Soares, 2001).

A cavidade interna e a espessura da polpa são variáveis relacionadas à qualidade e à pós-colheita dos frutos (Paiva et al., 2000). Os frutos com menor cavidade interna e maior espessura da polpa são desejados. Para a cavidade interna houve maior heterogeneidade entre os híbridos no segundo experimento com a formação de três grupos, tendo como destaque o híbrido Amarillo CP com menor média. Este resultado era esperado devido ao tamanho reduzido do fruto desse híbrido. As maiores cavidades foram observadas nos híbridos 
Tabela 2. Médias das características produtividade (PROD), peso médio do fruto (PM), cavidade interna (CI), espessura da polpa (EP), Aparências externa (AE) e interna (AI), perda de peso (PP), sólidos solúveis totais (SST) e firmeza da polpa (FP) de híbridos de melão cultivados no agropolo Mossoró-Assu, Experimento 2. Mossoró, ESAM, 2002.

\begin{tabular}{lccccccccc}
\hline Híbrido & $\begin{array}{c}\text { PROD } \\
\text { (Mg/ha) }\end{array}$ & PM (g) & Cl (cm) & EP (cm) & AE & Al & PP (\%) & SST (\%) & FP (N) \\
\hline Rochedo & $26,87 \mathrm{~b}$ & $1902 \mathrm{~b}$ & $6,78 \mathrm{~b}$ & $2,78 \mathrm{~b}$ & $3,04 \mathrm{~b}$ & $3,12 \mathrm{~b}$ & $4,89 \mathrm{a}$ & $9,24 \mathrm{a}$ & $28,93 \mathrm{~b}$ \\
Gold Pride & $28,37 \mathrm{~b}$ & $1654 \mathrm{c}$ & $6,43 \mathrm{~b}$ & $3,21 \mathrm{a}$ & $3,42 \mathrm{a}$ & $3,81 \mathrm{~b}$ & $2,67 \mathrm{c}$ & $9,05 \mathrm{a}$ & $31,05 \mathrm{~b}$ \\
Gold Mine & $25,41 \mathrm{~b}$ & $1632 \mathrm{c}$ & $7,17 \mathrm{a}$ & $2,85 \mathrm{~b}$ & $3,00 \mathrm{~b}$ & $3,83 \mathrm{~b}$ & $2,44 \mathrm{c}$ & $10,02 \mathrm{a}$ & $31,20 \mathrm{~b}$ \\
Gold Star & $29,25 \mathrm{~b}$ & $1687 \mathrm{c}$ & $7,70 \mathrm{a}$ & $2,99 \mathrm{a}$ & $2,67 \mathrm{~b}$ & $3,33 \mathrm{~b}$ & $1,98 \mathrm{~d}$ & $9,08 \mathrm{a}$ & $33,38 \mathrm{~b}$ \\
Amarillo C. P. & $19,54 \mathrm{c}$ & $950 \mathrm{e}$ & $4,41 \mathrm{c}$ & $2,41 \mathrm{~b}$ & $3,00 \mathrm{~b}$ & $3,11 \mathrm{~b}$ & $2,63 \mathrm{c}$ & $8,38 \mathrm{~b}$ & $40,05 \mathrm{a}$ \\
AFX 200 H & $32,54 \mathrm{a}$ & $1658 \mathrm{c}$ & $6,66 \mathrm{~b}$ & $3,39 \mathrm{a}$ & $3,00 \mathrm{~b}$ & $3,33 \mathrm{~b}$ & $4,54 \mathrm{a}$ & $9,17 \mathrm{a}$ & $29,83 \mathrm{~b}$ \\
Canarian K. & $23,65 \mathrm{~b}$ & $1987 \mathrm{~b}$ & $7,83 \mathrm{a}$ & $3,08 \mathrm{a}$ & $4,00 \mathrm{a}$ & $5,00 \mathrm{a}$ & $4,92 \mathrm{a}$ & $9,10 \mathrm{a}$ & $26,65 \mathrm{~b}$ \\
Tendency & $28,58 \mathrm{~b}$ & $2250 \mathrm{a}$ & $6,78 \mathrm{~b}$ & $2,96 \mathrm{a}$ & $2,50 \mathrm{~b}$ & $3,08 \mathrm{~b}$ & $3,80 \mathrm{~b}$ & $5,48 \mathrm{c}$ & $39,20 \mathrm{a}$ \\
Saturno & $32,90 \mathrm{a}$ & $1265 \mathrm{~d}$ & $6,73 \mathrm{~b}$ & $3,20 \mathrm{a}$ & $3,75 \mathrm{a}$ & $5,00 \mathrm{a}$ & $2,77 \mathrm{c}$ & $9,03 \mathrm{a}$ & $38,38 \mathrm{a}$ \\
Red Flesh & $32,54 \mathrm{a}$ & $1254 \mathrm{~d}$ & $6,55 \mathrm{~b}$ & $3,18 \mathrm{a}$ & $2,17 \mathrm{~b}$ & $2,42 \mathrm{~b}$ & $2,76 \mathrm{c}$ & $10,89 \mathrm{a}$ & $42,80 \mathrm{a}$ \\
AFX 700 H & $30,27 \mathrm{a}$ & $1542 \mathrm{c}$ & $6,25 \mathrm{~b}$ & $3,56 \mathrm{a}$ & $4,00 \mathrm{a}$ & $4,83 \mathrm{a}$ & $3,17 \mathrm{~b}$ & $11,20 \mathrm{a}$ & $38,40 \mathrm{a}$ \\
\hline Média & 28,66 & 1574,13 & 6,61 & 3,10 & 3,14 & 3,76 & 3,32 & 9,04 & 36,09 \\
CV\% & 28,42 & 17,26 & 4,32 & 10,22 & 19,03 & 18,06 & 23,49 & 7,88 & 11,42 \\
\hline
\end{tabular}

* Médias seguidas pela mesma letra nas colunas, não diferem, entre si, pelo teste de Scott-Knott, a 5\% de probabilidade.

Gold Mine, Gold Star e Canarian. No primeiro experimento, apenas o híbrido Supra, com a maior cavidade interna, diferiu dos demais.

Houve a formação de dois grupos em ambos os experimentos para espessura da polpa. Os destaques positivos, com maior espessura, no primeiro experimento, foram os híbridos Solarbel, Supra e Hy Mark. Os destaques negativos, menor espessura, no segundo experimento, foram os híbridos Rochedo, Gold Mine e Amarillo CP.

As aparências externa e interna são características fundamentais na vida útil pós-colheita do melão. Os frutos com notas inferior a 3,0 são comercializados no mercado externo (Gomes Junior et al., 2000). Com efeito, apenas o híbrido Imperial estaria descartado para a comercialização. Os principais motivos que conferiram perda de qualidade externa dos frutos foram manchas escuras, a fermentação e a queda do pedúnculo. A perda de qualidade interna deu-se principalmente devido ao amolecimento da polpa, sementes soltas e líquido na cavidade interna do fruto. Essas características foram observadas em outros trabalhos (Medeiros et al., 2001; Gomes Júnior et al., 2000).

Além de boas aparências interna e externa, é preciso que os frutos percam pouco peso durante o armazenamento, característica importante, uma vez que a comercialização é feita em unidade de massa (Menezes, 1996). Os híbridos DRG 1531 e DRG 1537, no experimento 1; e o híbrido Gold Star, no segundo experimento, destacaram-se com as menores perdas de peso. Essas perdas reduzem a qualidade do produto pelo enrugamento e o amolecimento do fruto. Os frutos dos híbridos GPS 400, Tendency, Imperial e Gold Star estavam extremamente enrugados e moles. Aspectos também observados em outros estudos de pós-colheita por Gomes Júnior $e t$ al. (2001) e Nascimento (2001).

Com relação ao teor de sólidos solúveis, verificou-se, no experimento 1 , que os híbridos DRG 1531 e DRG 1537 foram os de maiores estimativas, enquanto que os híbridos Amarillo CP e Tendency foram aqueles de menores médias. Uma presumível explicação para valores reduzidos de SST nestes dois híbridos foi a elevada severidade do oídio observada. O desenvolvimento do fungo na área foliar possivelmente tenha diminuído o processo fotossintético, reduzindo o valor do teor de sólidos solúveis. O teor de sólidos solúveis deve ser superior a $9 \%$ para ser comercializado. Nesse sentido, apenas dois híbridos alcançaram este valor no primeiro experimento. Por outro lado, aproximadamente $82 \%$ dos híbridos avaliados no segundo experimento estavam aptos para comercialização.

Segundo Filgueiras et al. (2000) a exigência mínima da firmeza da polpa no momento da colheita é de $22 \mathrm{~N}$. Assim sendo, a maior parte dos híbridos nos dois experimentos estavam acima do mínimo exigido. Os híbridos DRG 1531, DRG 1537, Galileu, Gold Star, Tendency, Saturno, Red Flesh e AFX 700 foram aqueles de maior firmeza de polpa.

Considerando as variáveis utilizadas na avaliação, constatou-se variação entre os híbridos nos dois experimentos. Entre os melões Gália, destacaram-se os híbridos DRG 1531 e DRG 1537. Os melões Red Flesh e AFX 700 foram os mais promissores entre os do tipo Orange Flesh. Por fim, entre os melões do tipo amarelo, destaques para Gold Mine, Gold Pride e Gold Star.

\section{LITERATURA CITADA}

DIAS, R.C. O agronegócio do melão no Nordeste: Análise prospectiva de sistemas naturais de cadeias produtivas. Brasília-DF: EMBRAPA/ DPD, 1998. $710 \mathrm{p}$.

FILGUEIRAS, H.A.C.; Colheita e manuseio póscolheita. In: FILGUEIRAS, H.A.C.; MENEZES, J.B.; ALVES, R.E. Melão pós-colheita: Brasília: EMBRAPA-SPI/FRUTAS DO BRASIL, 2000. p.23-41. (Frutas do brasil, 10).

GOMES JÚNIOR, J.; ARAUJO NETO, S.E.; MENEZES, J.B.; SIMÕES, A.N.; SILVA, G.G. Caracterização pós-colheita de melão "Piel de Sapo", genótipo Imara, sob condições ambientes. Revista Brasileira de Fruticultura, Jaboticabal, v.22, n. especial, p.32-36, 2000.

GOMES JÚNIOR, J. Armazenamento de dois genótipos de melão amarelo sob condições ambiente. Horticultura Brasileira, Brasília, v.19, n.1, p.42-49, 2001 
MEDEIROS, D.C.; GOMES JÚNIOR, J.; MENEZES, J.B.; SILVA, G.G. Vida útil pós-colheita de melão tipo Gália genótipo Solarking Fruticultura Brasileira, Jabotocabal, v.15, n.1, p.53-56, 2001.

MENEZES, J.B. Qualidade pós-colheita de melão tipo Gália durante a maturação e o armazenamento. Lavras: UFLA, 1996. (Tese doutorado).

MENEZES, J.B.; GOMES JÚNIOR, J; ARAÚJO, S.E.; SIMÕES, A.N. Amazenamento de dois genótipos de melão amarelo sob condições ambiente. Horticultura Brasileira, Brasília, v.19, n.1, p.42-49, 2001a.
MENEZES, J.B.; GOMES JUNIOR, J.; NUNES, G.H.; COSTA, F.B.; SOUZA, P.E. Qualidade póscolheita de melão tipo cantaloupe, colhido em dois estádios de maturação. Horticultura Brasileira, Brasília, v.19, n.3, p.356-360, 2001b.

NASCIMENTO, A.S. Armazenamento refrigerado de dois genótipos de melão amarelo 'Gold Mine' e 'Gold Pride' submetidos ao retardamento da colheita. 2001. 49 f. (Monografia graduação) ESAM, Mossoró-RN, 2001.
PAIVA, W.O.; NETO, H.S.; LOPES, A.G.S. Avaliação de linhagens de melão. Horticultura Brasileira, Brasília, v.18, n.2, p.109-113, 2000.

SILVA, E.M.F. Estudos sobre o mercado de frutas. FIPE, São Paulo, 1999. 373 p.

SOARES, S.P.F. Qualidade do melão (Cucumis melo L.) exportado pelo porto de Natal-RN. Mossoró: ESAM, 2001.(Monografia). 